\title{
Estudo dos transtornos cognitivos decorrentes da infecção pelo HIV-1
}

\author{
Study of cognitive dysfunction in HIV-1 \\ infected patients
}

\author{
Rosângela Souza KALIL' \\ Regina Maria Papais ALVARENGA' \\ Adilson José de ALMEIDA' \\ Carlos Alberto MORAIS-DE-SÁ'
}

\begin{abstract}
Resumo
A infecção pelo vírus da imunodeficiência humana atinge o Sistema Nervoso Central nos estágios iniciais, causando manifestações neuropsicológicas. Com o objetivo de estudar o desempenho de indivíduos infectados por este vírus em relação às funções cognitivas, foram avaliados 20 pacientes com contagem de linfócitos CD4+ acima de 200 células $/ \mathrm{mm}^{3}$, utilizando-se a Escala de Inteligência Wechsler para Adultos. Destes, cinco (25\%) eram do sexo masculino e 15 (75\%) do sexo feminino, com média de idade de 39,65 desvio-padrão de 10,15 anos. A contagem média de linfócitos CD4+ foi 467,20 desvio-padrão de 215,45 células $/ \mathrm{mm}^{3}$. Dentre quatorze pacientes que fizeram uso de terapia antirretroviral de alta atividade foi observado um caso com desempenho das funções cognitivas atenção e aprendizagem muito abaixo da média; os demais ( $n=13$ ) tiveram desempenho dentro da média. Por meio da Escala de Inteligência Wechsler para Adultos foi possível detectar desempenho abaixo do nível médio do funcionamento cognitivo em indivíduos com resultado positivo para vírus da imunodeficiência humana, mesmo em vigência de terapia antirretroviral de alta atividade.
\end{abstract}

Unitermos: Avaliação neuropsicológica. HIV. Transtornos cognitivos.

\begin{abstract}
In its early stages, HIV-1 infection involves the Central Nervous System causing neuropsychological manifestations. With the aim of studying the cognitive functions in HIV-1-infected patients, a total of 20 patients infected with HIV-1 with CD4+lymphocyte count $>200$ cells $/ \mathrm{mm}^{3}$ were studied in accordance with the Wechsler Adult Intelligence Scale (WAIS-III). The present study comprised 20 patients, 5 (25\%) male and 15 (75\%) female, with a mean age of 39.65 and standard deviation of 10.15 years. The mean CD4+ lymphocyte count was 467.20 with a standard deviation of 215.45 cells $/ \mathrm{mm}^{3}$. Amongst the 14 patients who received highly active anti-retroviral therapy (HAART), we noted 1 case in which the performance of the cognitive functions, attention span and learning, was considered to be very low according to WAIS-III, and the remaining $(n=13)$ patients who were treated with HAART demonstrated a performance that was not considered to be outside of the WAIS-III mean. By using WAIS-III testing, it was possible to detect performance of the cognitive functions considered to bevery low in HIV-1 infected individuals, even with those receiving HAART.
\end{abstract}

Uniterms: Neuropsychological assessment. Human-immunodeficiency-virus. Cognition disorders.

$\operatorname{crth}$

1 Universidade Federal do Estado do Rio de Janeiro, Hospital Universitário Gaffrée e Guinle, Clínica Médica B (10a Enfermaria). R. Mariz e Barros, 775, $2^{\circ}$ andar, Tijuca, 22270-004, Rio de Janeiro, RJ, Brasil. Correspondência para/Correspondence to: R. S. KALIL. E-mail: <kalilpsi92@gmail.com>. 
No curso natural da infecção pelo Vírus da Imunodeficiência Humana (HIV-1), o Sistema Nervoso Central (SNC) pode ser invadido pelo vírus precocemente, causando alterações do funcionamento cerebral (Clifford, 2002). OSNC é frequentemente acometido em aproximadamente 30\% dos indivíduos infectados (Grant et al., 1995). Estima-se que 40 milhões de pessoas vivam com HIV e/ou Síndrome da Imunodeficiência Adquirida (AIDS), incluindo crianças menores de 15 anos (World Health Organization, 2006). No Brasil, desde o início da década de 1980 até junho de 2006, o Ministério da Saúde registrou 433067 casos de AIDS (Simão, 2006).

Com o advento da terapia antirretroviral de alta atividade, ou Highly Active Antiretroviral Therapy (HAART), em 1996, as alterações cognitivas e neurológicas, em geral, sofreram considerável diminuição em sua frequência (Cook \& Tyor, 2006). Atualmente, pacientes tratados precocemente com esquemas combinados de medicamentos antirretrovirais mantêm-se clinicamente estáveis e, na maioria das vezes, sem componentes neurológicos detectáveis ao exame clínico rotineiro.

O diagnóstico de AIDS é estabelecido pela identificação de intercorrências clínicas tais como infecções oportunistas (pneumonia por Pneumocystis carinni, salmonelose sistêmica, tuberculose atípica, criptosporidíase, toxoplasmose, esofagite por cândida), diarreia recorrente, linfoma, sarcoma de Kaposi e lesões causadas pelo HIV-1 no SNC; e/ou laboratorialmente, quando a contagem de linfócitos T CD4+ encontra-se abaixo de 200 células/mm³ (Brew, 2004; Manji \& Miller, 2004).

A detecção precoce dos distúrbios neuropsicológicos pode ter importância no tratamento e prognóstico da doença. Os circuitos de consciência e memória, quando lesados precoce ou tardiamente, direta ou indiretamente pelo HIV-1, causam déficits funcionais. Na maioria das vezes, a infecção pelo HIV-1 no SNC ocorre de forma indireta, através dos monócitos do sangue circulante, para então infectar micróglia e astrócitos (Griffin, 1997).

Ao longo dos anos, surgem de forma lenta e progressiva manifestações clínicas discretas, como baixa capacidade de concentração, lentificação mental, apatia, baixa atividade psicomotora e modificações da memória verbal e não verbal, além da possibilidade de mudanças na personalidade (Paul, Cohen \& Stern, 2002; 466 Odiase, Ogurin \& Ogunniyi, 2007). Posteriormente, com o agravamento do processo, alterações mais significativas aparecem, como perdas expressivas de memória e afasia. A história natural das alterações neuropsicológicas na infecção por HIV-1 vem se modificando desde 1996 com o advento da HAART. Laboratorialmente, a contagem de células T CD4+ passou a ser um marcador da intensidade da imunodeficiência do paciente, além de bom sinalizador do tempo de duração da doença. $\bigcirc$ risco de manifestações neuropsicológicas aumenta na medida em que a contagem de células TCD4+ diminui, particularmente quando está abaixo de 200 células $/ \mathrm{mm}^{3}$, sendo, portanto, um fator indicativo de possíveis distúrbios cognitivos (Brew, 2004; Manji \& Miller, 2004).

Não obstante a boa resposta à HAART, deficiências fixas resultantes de perdas neurais anteriores não podem ser recuperadas e, portanto, alguns eventuais déficits cognitivos prévios não melhoram. Por outro lado, a HAART pode não interferir nas formas de evolução crônica de alterações neuropsicológicas. A ativação de monócitos, da micróglia e dos astrócitos, ao manter produtiva a infecção pelo HIV-1 no cérebro, pode, ao mesmo tempo, ser responsável pelos danos já impostos ou até pela proteção de neurônios não lesados. Assim, o aumento da sobrevida ocasionado pela HAART, além de estabilizar ou diminuir inicialmente os distúrbios cognitivos, poderá até proporcionar, a partir de maior tempo de sobrevida do doente, um período maior para que o HIV-1 possa lesar o SNC e promover danos clínicos de aparecimento tardio (Brew, 2004).

Os testes neuropsicológicos têm possibilitado, na população de indivíduos HIV-1+', a identificação de função cognitiva intacta, além de alterações mensuráveis de disfunções precoces tardias ou recidivadas leves, moderadas ou intensas (Ashford et al., 2007).

O déficit cognitivo pode ser responsável pela menor adesão ao tratamento da infecção pelo HIV-1, antecipando um quadro de doenças oportunistas (Miller et al., 1990), bem como produzindo falha no uso regular de preservativos. A presença do HIV-1 no SNC pode ser a principal responsável pelas manifestações neuropsicológicas e por alterações na qualidade de vida do indivíduo (Hinkin et al., 2002; Mehan \& Brush, 2001; Odiase, Ogurin \& Ogunniyi, 2007). Estas ocorrem como distúrbios de diversos graus nas áreas motora, comportamental e cognitiva (Vitiello et al., 2007). 
Nos estágios precoces do complexo demencial da AIDS, os resultados de exames neurológicos podem ser normais (Cohen, Perlstein \& Braver, 1997; MacArthur et al., 2003). Quando presentes, as anormalidades são poucas e sutis, caracterizando formas leves de danos cognitivos associados ao HIV-1. Embora déficits cognitivos associados ao HIV-1 raramente se desenvolvam em pacientes imunocompetentes, estudos revelaram a presença de declínios cognitivos em pacientes HIV-1 positivos assintomáticos e suas implicações na vida profissional (Koralnik et al., 1990; Newman, Lunn \& Harrison, 1995).

As funções cognitivas mais afetadas nos estágios iniciais da AIDS são:

- Memória: estudos realizados utilizando métodos de imagem demonstraram o aumento da atividade cerebral durante tarefas relacionadas à memória de trabalho em pacientes HIV-1+ ${ }^{+}$com demência leve (Grant et al., 1987; Odiase, et al., 2007).

- Aprendizagem: relatos de estudos apontam para a redução nos escores de testes que medem aprendizagem verbal (Grant et al., 1987; Tozzi et al., 2001).

- Atenção e concentração: a atenção e a concentração podem ser reduzidas pelo HIV-1, com lentificação do processamento da informação e das respostas, resultando em desatenção e distração (Martin et al., 1992).

- Velocidade psicomotora: é influenciada por fatores como piora clínica, complicações infecciosas, uso de medicações e, não infrequentemente, piora da eficiência mental, atingida pela depressão, preocupação e ansiedade (Waldrop-Valverde, Ownby, Wilkie, Mack, Kumar \& Metsch, 2006).

- Velocidade do processamento da informação e pensamento: a lentificação do pensamento acompanhada de redução na velocidade do processamento da informação é uma característica importante dos efeitos cognitivos da infecção pelo HIV-1 (Wilkie, Eisdorfer, Morgan, Loewenstein \& Szapocznik, 1990; Martin et al., 1992).

Distúrbios psiquiátricos são frequentemente associados à AIDS; o mais encontrado é a depressão. Esta pode ser confundida com quadros cogitivo-demenciais pela semelhança de sintomas, como transtornos da motivação e do humor (Seth, Granville-Grossman, Goldmeir \& Lynh, 1991).
Assim, o objetivo deste trabalho foi estudar o desempenho de indivíduos infectados pelo HIV-1 em relação às funções cognitivas, utilizando-se a Escala de Integência Wechsler para Adultos (WAIS-III).

\section{Método}

Estudo prospectivo, observacional, com delineamento transversal.

\section{Participantes}

Durante o período de fevereiro de 2006 a outubro de 2006 foram atendidos 216 pacientes com HIV/AIDS no Ambulatório de Imunologia e Alergia da Clínica Médica B do Hospital Universitário Gaffrée e Guinle da Universidade Federal do Estado do Rio de Janeiro (UNIRIO). Os pacientes estudados tiveram confirmação da infecção pelo HIV-1 por dois testes de ensaio imunoenzimático (Enzyme-Linked Immunosorbent Assay ou ELISA-Vidas ${ }^{\circledR}$ HIV DUO Ultra, Biométieux ${ }^{\circledR}$ AS, Marcy I'Etoile, França; Access+ HIV-1/2, New, Bio-Rad, Marnesla-Coquette, França), e um Western Blot (New Lav Blot I, Bio-Rad, Marnes-la-Coquette, França), realizados no Laboratório de Pesquisa em Imunologia e AIDS (LAPIA) da Clínica Médica B.

Também foram realizadas as contagens de linfócitos $\mathrm{T} \mathrm{CD4}^{+}$por citometria de fluxo utilizando-se o reagente BD FACSCount ${ }^{\mathrm{TM}}$ Controls (BD Biosciences Immunocytometry Systems, San Jose, Califórnia, Estados Unidos da América) e a determinação da carga viral do HIV-1 pelo método baseado na ampliação das sequências de ácidos nucleicos (Nucleic Acid Amplification Based Assay ou NASBA) (NucliSens ${ }^{\circledR}$ HIV-1 QT, Biomérieux, Boxtel, Holanda).

Foram adotados os seguintes critérios de inclusão: 1) indivíduos alfabetizados, com 18 anos de idade ou mais; 2) indivíduos com infecção por HIV-1; 3) indivíduos apresentando contagem de linfócitos T CD4 ${ }^{+}$ acima de 200 células $/ \mathrm{mm}^{3}$.

Foram adotados os seguintes critérios de exclusão: 1) gestantes; 2) indivíduos com idade acima de 65 anos; 3) usuários de drogas endovenosas; 4) portadores de psicoses primárias; 5) pacientes que estivessem usando medicamentos causadores de efeitos adversos 
ou psiquiátricos sobre o SNC, tais como efavirenz, benzodiazepínicos e outros agentes de ação psicoativa; 6) indivíduos com doenças oportunistas, especialmente do SNC, em atividade ou não; 7) não concordância com o Termo de Consentimento Livre e Esclarecido".

A seleção dos pacientes foi realizada em três etapas consecutivas:

Primeira etapa: foram consultados os prontuários médicos de 216 pacientes, a partir dos quais foram analisados os critérios de inclusão e exclusão. Deste total foram excluídos 166 indivíduos, permanecendo 50 pacientes para o estudo. Os pacientes foram excluídos devido aos seguintes fatores: infecção por HIV-1 no SNC ( $n=60)$; uso de efavirenz ( $n=52)$; contagem de células $T$ $\mathrm{CD}^{+}$abaixo de 200 células $/ \mathrm{mm}^{3}(\mathrm{n}=34)$; idosos ( $\left.\mathrm{n}=11\right)$; não concordância com o Termo de Consentimento Livre e Esclarecido" ( $n=7)$; analfabetos ( $n=2)$.

Segunda etapa: foi realizada a marcação de consultas para os pacientes que atenderam aos critérios de seleção ( $n=50)$. Foram então excluídos 30 pacientes, por não terem comparecido à consulta.

Terceira etapa: esta etapa foi caracterizada pela avaliação neuropsicológica dos 20 indivíduos HIV-1 positivos.

\section{Instrumentos}

Os pacientes foram submetidos aos seguintes instrumentos:

a) Anamnese: para coleta de dados de identificação, endereço, doenças pré-existentes, medicamentos, exames complementares e funcionamento cognitivo.

b) Inventários de Beckpara Ansiedadee Depressão: instrumentos autoaplicáveis com duração de aproximadamente dez minutos.

c) Doze subtestes da Escala de Inteligência de Wechsler para Adultos ou Wechsler Adult Intelligence Scale (WAIS-III): instrumento clínico de aplicação individual, com duração de aproximadamente 120 minutos, que permite a avaliação de ampla gama de domínios relacionados às alterações cognitivas causadas pelo HIV-1 no SNC. Trata-se de uma escala prática, investigativa, adaptada e, sobretudo, normatizada à realidade brasileira (Wechsler, 2004). Apresenta 12 subtestes de ava-

468 liação do funcionamento intelectual:
I) Completar figuras: avalia a acuidade visual, o raciocínio, o julgamento e a memória de longo prazo. São apresentadas 25 figuras coloridas, faltando partes importantes. O examinando deve indicar a parte importante que está faltando em cada figura dentro de um tempo limite de 20 segundos.

II) Vocabulário: avalia a linguagem (habilidade verbal, desenvolvimento da linguagem e conhecimento das palavras). O examinando define, oralmente, o significado de cada palavra apresentada em uma lista de 33 palavras lidas, em voz alta, pelo examinador.

III) Códigos: avalia o desempenho psicomotor, a atenção seletiva, a atenção sustentada, a velocidade de resposta, a organização visomotora envolvendo velocidade e acurácia na coordenação olho/mão e a memória visual de curto prazo. O examinando deve identificar e copiar símbolos que estão associados aos números dispostos em um modelo. A pontuação é determinada pelo número de símbolos copiados corretamente em 120 segundos.

IV) Semelhanças: avalia as funções executivas (comparações verbais abstratas, formação de conceitos e habilidade verbal geral). É composto por 19 itens contendo duas palavras que representam objetos ou conceitos comuns que são apresentados, oralmente, ao examinando. Este deve identificar a similaridade entre dois objetos ou conceitos ou qual a característica comum entre eles.

V) Cubos: avalia o componente espacial da percepção em um nível conceitual e a habilidade construtiva (construção). Avalia a organização perceptual e visual, a conceitualização abstrata (análise do todo em suas partes componentes) e a formação de conceito não verbal e visualização espacial. Contém nove cubos; cada cubo tem dois lados brancos, dois vermelhos e dois metade vermelho, metade branco. O examinando deve reproduzir modelos de duas cores. Cada modelo tem um tempo determinado para a construção.

VI) Aritmética: avalia habilidades de cálculo e raciocínio matemático, como capacidade de resolver as quatro operações básicas, habilidade de resolução de problemas complexos, atenção, abstração, concentração, flexibilidade cognitiva e memória imediata. 0 examinando é submetido a 20 problemas de aritmética que ele deve resolver mentalmente e responder oralmente dentro de um tempo limite. 
VII) Raciocínio matricial: avalia o processamento de informações visuais e raciocínio abstrato envolvendo quatro itens: padrões contínuos e discretos, classificação, raciocínio analógico e raciocínio em série. Cada uma das 26 figuras consiste em uma matriz-estímulo, na qual falta uma parte, e cinco alternativas de resposta. 0 examinando seleciona a resposta correta que completa a matriz, não havendo limite de tempo para sua execução.

VIII) Dígitos: avalia a capacidade de armazenamento de curto prazo (recordação e repetição imediata). Representa dois elementos básicos da atenção: rapidez e span (quantidade do sistema de processamento). É apresentada, oralmente, ao examinando, uma série de números, que deve ser repetida literalmente na ordem direta e na ordem inversa. Não há limite de tempo.

IX) Informações: avalia memória retrógrada, memória semântica e habilidade verbal geral (aquisição de conhecimento e retenção). Pede-se ao examinando que responda, oralmente, a uma série de 28 questões que se relacionam aos eventos comuns, objetos, lugares e pessoas, não havendo limite de tempo para sua realização.

X) Arranjo de figuras: avalia o raciocínio sequencialmente ordenado sobre material visual. Visa reconhecer a essência da história e antecipar e compreender a sequência de eventos, particularmente eventos sociais; esta habilidade está, portanto, envolvida na capacidade de antecipar consequências, planejar e elaborar conceitos temporais.

XI) Compreensão: avalia o raciocínio verbal, a memória remota, o julgamento, a abstração e a flexibilidade cognitiva (avaliação e uso de experiências passadas e conhecimento dos padrões convencionais de comportamento). Solicita-se ao examinando que responda oralmente a uma série de 18 questões que exigem soluções para problemas cotidianos ou o entendimento de regras e conceitos sociais. O tempo de execução da tarefa não é considerado.

XII) Procurar símbolos: avalia atenção, memória de curto prazo, rapidez de processamento, precisão, discriminação perceptual e habilidade para explorar símbolos visuais. Para cada item deste subteste, o examinando analisa, visualmente, dois grupos de sím- bolos: um grupo modelo (composto de dois símbolos) e um grupo de procura (composto de cinco símbolos), e indica se um dos símbolos faz parte do grupo de procura. O examinando responde ao maior número de itens possíveis dentro de um limite de 120 segundos.

Para a obtenção dos índices fatoriais foi considerada a soma dos subtestes:

- Índice de Compreensão Verbal (ICV): vocabulário + semelhanças + informação.

- Índice de Organização Perceptual (IOP): completar figuras + cubos + raciocínio matricial.

- Índice de Memória Operacional (IMO): aritmética + dígitos.

- Índice de Velocidade do Processamento (IVP): códigos + procurar símbolos.

Os instrumentos citados foram aplicados pelo pesquisador principal. O estudo foi aprovado pelo Comitê de Ética em Pesquisa do Hospital Universitário Gaffrée e Guinle (protocolo n¹22/2005).

Os dados coletados referentes à anamnese e aos resultados de exames complementares e da bateria de subtestes foram codificados com números e armazenados em banco de dados criado no programa Access 97 (versão 6.0). Com base na análise univariada (estatística descritiva), foram determinados os valores para média, desvio-padrão, mediana, faixa de variação, amplitude, frequência e percentis $\left(5^{\circ}\right.$ a $\left.95^{\circ}\right)$. A análise estatística foi realizada utilizando-se o pacote Statistical Package for the Social Sciences (SPSS) for Windows, release 12.0 .

\section{Resultados}

\section{Características demográficas, clínicas e laboratoriais da amostra estudada}

Foram avaliados 20 indivíduos: cinco (25\%) do sexo masculino e 15 (75\%) do sexo feminino, com média de idade de 39,65, desvio-padrão de 10,15 anos (variando de 24 a 62 anos). Com relação ao estágio da infecção pelo HIV-1, não houve observação de qualquer caso com alteração neurológica clinicamente detectável. A contagem média de células T CD4+ foi de 467,20 células $/ \mathrm{mm}^{3}$, variando de 202,00 a 977,00 células/mm³. Entre 
os pacientes estudados, seis (30\%) não fizeram uso de HAART e 14 (70\%) relataram uso de HAART.

\section{Escores dos subtestes}

Com relação aos escores dos subtestes (Tabela 1), a mediana e a faixa de variação foram as seguintes: compreensão de figuras=12,00 pontos (8,00 a 15,00 pontos); vocabulário=9,00 pontos (5,00 a 12,00 pontos); códigos $=11,00$ pontos (7,00 a 14,00 pontos); semeIhanças $=11,00$ pontos (8,00 a 15,00 pontos); cubos $=11,00$ pontos (6,00 a 12,00 pontos); aritmética $=8,50$ pontos (7,00 a 13,00 pontos); raciocínio matricial $=9,00$ pontos (7,00 a 16,00 pontos); dígitos=10 pontos (8,00 a 16,00 pontos); informações=9,50 pontos (7,00 a 13,00 pontos); arranjo de figuras $=11,00$ pontos (6,00 a 17,00 pontos); compreensão=9,00 pontos (4,00 a 13,00 pontos); procurar símbolos=11,50 pontos (6,00 a 16,00 pontos).

Foram observados dois resultados abaixo do percentil 5\% em relação aos subtestes cubos e informações, dois resultados acima do percentil 95\% em relação aos subtestes dígitos e arranjo de figuras, um resultado com valor abaixo do percentil 5\% e outro acima do percentil 95\% para o subteste procurar símbolos.

\section{Índices fatoriais}

Oíndice de compreensão verbal mede o conhecimento adquirido, o raciocínio verbal e a atenção a materiais verbais, sendo o índice fatorial aquele que reflete uma medida mais refinada da compreensão verbal. $\mathrm{O}$ índice de organização perceptual é uma medida mais refinada de raciocínio fluido, não verbal, de atenção a detalhes e de integração visomotora, mais especificamente a solução de problemas visuoespaciais. O índice de memória operacional reflete a capacidade do indivíduo de prestar atenção, reter as informações, processá-las na memória por um breve período e, por último, formular a resposta. O índice de velocidade de processamento reflete a capacidade do indivíduo de processar informações visuais rapidamente.

Com relação aos escores dos índices fatoriais (Tabela 2), a mediana e a faixa de variação foram as seguintes: índice de compreensão verbal=29,00 pontos (19,00 a 37,00 pontos); índice de organização perceptual=31,50 pontos (21,00 a 42,00 pontos); índice de memória operacional=19,00 pontos $(15,00$ a 24,00 pontos); índice de velocidade de processamento $=22,50$ pontos (13,00 a 28,00 pontos);

$\mathrm{Na}$ amostra estudada foi observado um caso (paciente n 5), com IVP considerado muito inferior de acordo com a classificação de WAIS-III (Figura 1). Os demais pacientes $(n=17)$ apresentaram desempenho dentro da média em relação aos índices fatoriais.

\section{Discussão}

Com a introdução da HAART, os indivíduos com HIV/AIDS apresentaram redução da incidência de infecções oportunistas, de tumores associados ao HIV-1,

Tabela 1. Análise univariada dos parâmetros dos subtestes de uma amostra ( $n=20)$ de pacientes com infecção pelo HIV. Rio de Janeiro (RJ), 2006.

\begin{tabular}{|c|c|c|c|c|c|c|c|c|c|c|c|c|c|}
\hline & \multirow{2}{*}{$\begin{array}{l}\text { Estimativas dos } \\
\text { parâmetros pontos) }\end{array}$} & \multicolumn{12}{|c|}{ Subtestes de WAIS-III } \\
\hline & & CF & VOC & COD & SEM & CLUB & ARI & $\mathrm{RM}$ & DIG & INF & AF & $\mathrm{COM}$ & PS \\
\hline & Média & 11,85 & 9,50 & 11,15 & 10,95 & 10,25 & 9,05 & 10,70 & 10,10 & 9,45 & 11,05 & 9,00 & 11,35 \\
\hline & Desvio-padrão & 2,03 & 2,09 & 2,00 & 1,57 & 1,68 & 1,90 & 3,27 & 1,98 & 1,95 & 2,83 & 2,51 & 2,51 \\
\hline & Mediana & 12,00 & 9,50 & 11,00 & 11,00 & 11,00 & 8,50 & 9,00 & 10,00 & 9,50 & 11,00 & 9,00 & 11,50 \\
\hline & Moda & 12,00 & 12,00 & 12,00 & 12,00 & 11,00 & 8,00 & 9,00 & 9,00 & 7,00 & 11,00 & 11,00 & 12,00 \\
\hline ק & Valor mínimo & 8,00 & 5,00 & 7,00 & 8,00 & 6,00 & 7,00 & 7,00 & 8,00 & 7,00 & 6,00 & 4,00 & 6,00 \\
\hline 西 & Valor máximo & 15,00 & 12,00 & 14,00 & 15,00 & 12,00 & 13,00 & 16,00 & 16,00 & 13,00 & 17,00 & 13,00 & 16,00 \\
\hline$\stackrel{\text { 至 }}{=}$ & Amplitude & 7,00 & 7,00 & 7,00 & 7,00 & 6,00 & 6,00 & 9,00 & 8,00 & 6,00 & 11,00 & 9,00 & 10,00 \\
\hline$\stackrel{D}{+}$ & Percentil 5\% & 8,00 & 5,05 & 7,05 & 8,05 & 6,10 & 7,00 & 7,00 & 8,00 & 7,00 & 6,05 & 4,05 & 6,15 \\
\hline$\stackrel{\varrho}{=}$ & Percentil 95\% & 15,00 & 12,00 & 14,00 & 14,85 & 12,00 & 13,00 & 16,00 & 15,00 & 13,00 & 16,95 & 12,90 & 15,90 \\
\hline
\end{tabular}

"WAIS: Wechsler adult intelligence scale ou escala de inteligência de Wechsler para adultos; CF: compreensão de figuras; VOC: vocabulário; COD: códigos; SEM: semelhanças; CUB: cubos; ARI: aritmética; RM: raciocínio matricial; DIG: dígitos; INF: informações; AR: arranjo de figuras; COP: 
Tabela 2. Análise univariada dos parâmetros dos índices fatoriais de uma amostra ( $n=20)$ de pacientes com infecção pelo HIV. Rio de Janeiro, 2006.

\begin{tabular}{|c|c|c|c|c|}
\hline \multirow{2}{*}{$\begin{array}{l}\text { Estimativas dos } \\
\text { parâmetros (pontos) }\end{array}$} & \multicolumn{4}{|c|}{ Índices fatoriais* } \\
\hline & ICV & IOP & $\mathrm{IMO}$ & IVP \\
\hline Média & 28,60 & 1,40 & 19,20 & 22,00 \\
\hline Desvio-padrão & 5,69 & 5,58 & 2,44 & 3,44 \\
\hline Mediana & 28,60 & 31,00 & 19,00 & 22,50 \\
\hline Moda & 35,00 & 28,00 & 18,00 & 23,00 \\
\hline Valor mínimo & 19,00 & 21,00 & 15,00 & 13,00 \\
\hline Valor máximo & 37,00 & 42,00 & 24,00 & 28,00 \\
\hline Amplitude & 18,00 & 21,00 & 9,00 & 15,00 \\
\hline Percentil 5\% & 19,05 & 21,00 & 15,00 & 13,25 \\
\hline Percentil 95\% & 36,95 & 41,00 & 23,95 & 27,95 \\
\hline
\end{tabular}

* Índices Fatoriais de WAIS (Wechsler Adult Intelligence Scale ou Escala de Inteligência de Wechsler para Adultos).

ICV: índice de compreensão verbal; IOP: índice de organização perceptual; IMO: índice de memória operacional; IVP: índice de velocidade de processamento.

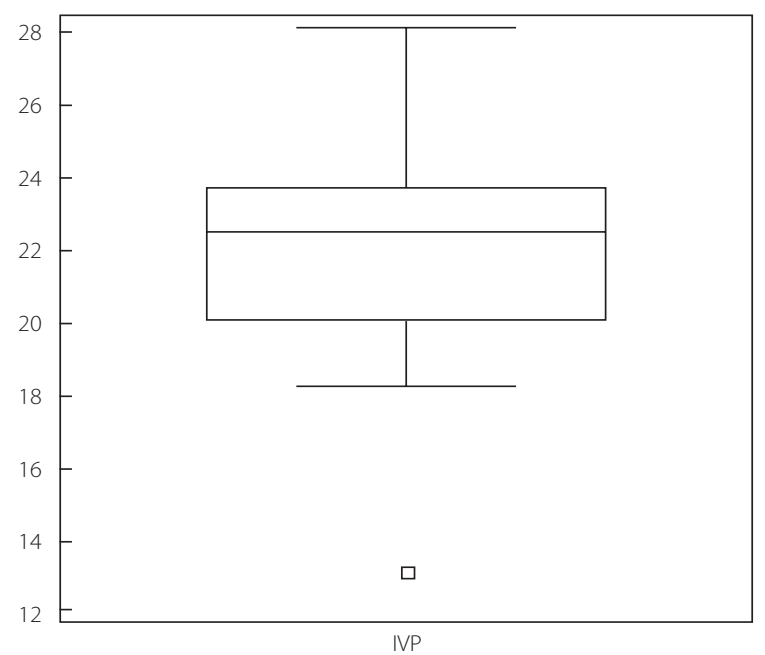

Figura 1. Diagrama de caixas do índice de velocidade do processamento (IVP) mostrando um escore considerado muito inferior (abaixo do $5^{\circ}$ percentil) do caso $n^{\circ} 5$.

Nota: A caixa central representa os valores do primeiro quartil $\left(25^{\circ}\right)$ ao terceiro quartil $\left(75^{\circ}\right)$ e a mediana ( 22,5 pontos) é mostrada como uma linha média horizontal.

bem como diminuição acentuada da demência associada ao HIV-1 (Brodt, Kamps, Gute, Knupp, Staszewski \& Helm, 1999). Os padrões de alterações neuropsicológicas foram acentuadamente diferentes quando se comparou a era pré-HAART com a HAART. Houve nítida diminuição de manifestações neuropsicológicas na era pós-HAART, particularmente com referência ao aprendizado e à atenção, fluência verbal e visuoconstrução. No entanto, no que se refere ao aprendizado e à atenção, foi observada uma deterioração dessas funções cognitivas (Cysique, Maruff \& Brew, 2004).

Neste estudo, embora tenham sido observados resultados fora da média em uma proporção dos subtestes utilizados, foram obtidas, mediante a análise dos índices fatoriais, medidas mais específicas das funções cognitivas. Desta forma, em comum com os achados das fontes pesquisadas, observou-se um caso de paciente do sexo masculino, com 39 anos, com contagem de células T CD4 ${ }^{+}$de 366 células $/ \mathrm{mm}^{3}$, em uso da combinação terapêutica lamivudine + atazanavir + ritonavir, que apresentou desempenho abaixo da média (considerado muito inferior na classificação dos escores da WAIS-III) em atenção e aprendizado, podendo sugerir uma deficiência no funcionamento cognitivo. Este achado não pode ser considerado perda ou alteração relacionada à HAART, já que não foram realizadas comparações anteriores desse mesmo paciente (teste e reteste).

Déficits neuropsicológicos típicos, como perda da atenção sustentada, flexibilidade mental, velocidade motora, memória (Miller et al., 1990) e memória de trabalho (Law et al., 1994; Grassi et al., 1999), podem ser detectados em indivíduos com HIV/AIDS quando se aplicam testes neuropsicológicos adequados (Chang, Ernest, Leonildo-Yee, Walot \& Singer, 1999; Suwanwelaa et al., 2000). Estes dados não puderam ser confirmados no estudo aqui descrito, provavelmente pela necessi- 
dade de ampliação do número de casos estudados e um período mais prolongado entre a infecção e o tratamento. É possível que, em estudos futuros, incluindo a retestagem neuropsicológica, possam ser encontrados tais indícios.

Embora alguns autores (Chang et al., 1999; Suwanwelaa et al., 2000) tenham relatado que alterações cognitivas possam ser mais facilmente detectadas por meio de exames de imagens em indivíduos com lesões mínimas ou mesmo imperceptíveis, estudo mais recente (Kemppainen, 2004) revelou que os testes neuropsicológicos são ferramentas mais sensíveis na identificação destas alterações, além de acessíveis quanto aos custos de execução e à simplicidade de aplicação, diferentemente dos exames de neuroimagem. A bateria de testes cognitivos tem a capacidade de mostrar a função cerebral fora do repouso, em plena atividade. Convém ressaltar que, para um país emergente como o Brasil, é possivel implantar estes testes em larga escala em qualquer município.

Os resultados deste estudo indicam que é possível detectar desempenho abaixo do nível médio do funcionamento cognitivo por meio da Escala de Inteligência Wechsler para Adultos (WAIS-III) em indivíduos infectados pelo HIV-1, mesmo em vigência de tratamento com HAART.

\section{Referências}

Ashford, J. W., Borson, S., O'Hara, R., Dash, P., Frank, L., Robert P., et al. (2007). Should older adults be screened for dementia? It is important to screen for evidence of dementia! The Journal of the Alzheimer's Association, 3 (2), 75-80.

Brasil. Ministério da Saúde. (2004). Critérios de definição de casos de AIDS em adultos e crianças. Série Manuais n 60. Brasília: MS.

Brew, B. J. (2004). Evidence for a change in AIDS dementia complex in the era of highly active antiretroviral therapy and possibility of new a forms of AIDS dementia complex. AIDS, 18 (Suppl 1), S75-S70.

Brodt, H. R., Kamps, B. S., Gute P., Knupp, B., Staszewski, S., \& Helm, E. B. (1997). Changing incidence of AIDS-defining illness in the era of antiretroviral combination therapy. AIDS, 11 (14), 1731-1739.

Chang, L., Ernest, T., Leonildo-Yee, M., Walot, I., \& Singer, E. (1999). Cerebral metabolite abnormalities correlate with clinical severity of HIV-cognitive motor complex. Neurology, 52 (1), 100-108.

Clifford, D. B. (2002). AIDS dementia. The Medical Clinics of 472 North America, 86 (3), 537-550.
Cohen, J. D., Perlstein, W. M., \& Braver, T. S. (1997). Temporal dynamics of brain activation during a working memory task. Nature, 386 (6625), 604-608.

Cook, J., \&Tyor, W. (2006). The patogenesis of HIV-associated dementia: recent advances using a SCID mouse model of dementia of HIV-encephalitis. The Einstein Journal of Biology and Medicine, 22 (1), 32-40.

Cysique, L.A., Maruff, P., \& Brew, B.J. (2004). Prevalence and pattern of neuropsychological impairment in human immunodeficiency vírus-infected/acquired across pre and pos-highly active antiretroviral therapy eras: a combined study of two cohorts. Journal of Neurovirology, $10(6), 350-357$.

Grant, I., Atkinson, J. H., Hesselink, J. R., Kennedy, C. J., Richman, D. D., Spector, S. A., et al. (1987). Evidence for early central nervous system involvement in the acquired immunodeficiency syndrome (AIDS) and other HIV infection. Studies with neuropsychological testing and magnetic resonance imaging. Annals of Internal Medicine, 107 (6), 828-836

Grassi, B., Graghebtini, G., Campana, A., Grassi, E., Bertelli, S., Cinque, P., et al. (1999). Spatial working memory in asymptomatic HIV-infected subjects. The Journal of Neuropsychiatry and Clinical Neurosciences, 11 (3), 387-391.

Griffin, D. E. (1997). Cytokines in the brain during viral infection: clues to HIV-associated dementia. The Journal of Clinical Investigation, 100 (12), 2948-2951.

Hinkin, C. H., Castellon, A. A., Durvasula, R. S., Hardy, D. J., Lam, M. N., Mason, K. I., et al. (2002). Medication adherence among HIV+ adults: effect of cognitive dysfunction and regimen complexity. Neurology, 59 (12), 1944-1950.

Kemppainen, J. K. (2004). Assessment of cognitive changes in HIV/AIDS. Neurology Today, 4 (11), 66-70.

Koralnik, I. J., Beaumanoir, A., Häusler, R., Kohler, A., Safran, A. B., Delacoux, R., et al. (1990). Controlled study of early neurological abnormalities in men with asymptomatic HIV infection. The New England Journal of Medicine, 323 (13), 864-870.

Law, W. A., Martin, A., Mapou, R. L., Roller, T. L., Salazar, A. M., Temoshok, L. R., et al. (1994). Working memory in individuals with HIV infection. Journal of Clinical and Experimental Neuropsychology, 16 (2), 173-182.

Lezak, M. D. (2004). Neuropsychological assessment. New York: Oxford University.

McArthur, J. C., Haughey, N., Gartner, S., Conant, K., Pardo, C., Nath, A., et al. (2003). Human immunodeficiency virus-associated dementia: an envolving disease. Journal of Neurovirology, 9 (2), 205-221.

Manji, H., \& Miller, R. (2004). The neurology of HIV infection. Journal of Neurology, Neurosurgery, and Psychiatry, 5 (Suppl 1), i29-35.

Martin, E. M., Robertson, L. C., Edelstein, H. E., Jagust, W. J., Sorensen, D. J., San Giovanni, D., et al. (1992). Performance of patients with early HIV-1 infection on the stroop task. Journal of Clinical and Experimental Neuropsychology, 14 (5), 3311-3320. 
Meehan, R. A., \& Brush, J. A. (2001). An overview of AIDS dementia complex. American Journal of Alzheimer's Disease and other Dementias, 16 (4), 225-229.

Miller, E. N., Selnes, O. A., McArthur, J. C., Satz, P., Becker, J. T., Cohen, B. A., et al. (1990). Neuropsychological performance in HIV-1 infected homossexual men: the multicenter AIDS cohort study (MACS). Neurology, 40 (2), 197-203.

Newman, S. P, Lunn, S., \& Harrison, M. J. C. (1995). Do asymptomatic HIV seropositive individuals show cognitive deficit? AIDS, 9 (11), 1211-1220.

Odiase, F. E., Ogurin, O. A., \& Ogunniyi, A. A. (2007). Memory performance in HIV/AIDS- a prospective case control study. The Canadian Journal of Neurological Sciences, 34 (2), 154-159.

Paul, R. H., Cohen, R. A., \& Stern, R. A. (2002). Neurocognitive manifestations of human immunodeficiencyvirus. CNS Spectrums, 7 (12), 860-866.

Sacktor, N., Lyles, R. H., Skolasky, R., Kleeberger, C., Selnes, O. A., Miller, E. N., et al. (2001). HIV-associated neurologic disease incidence changes. Multicenter AIDS cohort study, 1990-1998. Neurology, 56 (2), 257-260.

Seth, R., Granville-Grossman, K., Goldmeier, D., \& Lynch, S. (1991). Psychiatric illnesses in patients whit HIV infection and AIDS referred to the liaison psychiatrist. The British Journal of Psychiatry, 159 (2), 347-350

Simão, M. (2006). Apresentação. Boletim Epidemiológico-AIDS e DST, 3 (1) 3-6.

Suwanwelaa, N., Phanuphak, P., Phanthumchinda, K., Suwanwela, N. C., Tantivatana, J., Ruxrungtham, K., et al.
(2000). Magnetic resonance spectroscopy of the brain in neurologically asymptomatic HIV-infected patients. Magnetic Resonance Imaging, 18 (7), 859-865.

Tozzi, V., Balestra, P., Galgani, S., Narciso, P., Sampaolesi, A., Antinori, A., et al. (2001). Changes in neurocognitive performance in cohort of patients treated with HAART for 3 years. Journal of Acquired Immune Deficiency Syndromes (1999), 28 (1), 19-27.

Vittiello, B., Goodkin, K., Ashtana, D., Shapshak, P., Atkindon, J. H., Helseltine. P. N., et al. (2007). HIV-1 RNA concentration and cognitive performance in a cohort of HIV-positive people. AIDS, 21 (11), 1415-1422.

Waldrop-Valverde, D., Ownby, R. L., Wilkie, F. L., Mack, A., Kumar, M., \& Metsch L. Neurocognitive aspects of medication adherence in HIV-positive injecting drug users. AIDS and Behavior, 10 (3), 287-297.

Wechsler, D. (2004). Escala de inteligência para adultos: manual para administração e avaliação. São Paulo: Casa do Psicólogo.

Wilkie, F. L., Eisdorfer, C., Morgan, R., Loewenstein, D. A., \& Szapocznik, J. (1990). Cognition in early human immunodeficiency virus infection. Archives of Neurology, 47 (4), 433-440.

World Health Organization. (2006). Global estimates for adults and children. Geneva: WHO.

Recebido em: 5/9/2007

Versão final reapresentada em: 12/5/2008

Aprovado em: 25/6/2008 